PROCEEDINGS OF THE

AMERICAN MATHEMATICAL SOCIETY

Volume 135, Number 12, December 2007, Pages 3847-3856

S 0002-9939(07)08912-5

Article electronically published on September 10, 2007

\title{
A COMPUTER PROOF OF MOLL'S LOG-CONCAVITY CONJECTURE
}

\author{
MANUEL KAUERS AND PETER PAULE
}

(Communicated by Jim Haglund)

\begin{abstract}
In a study on quartic integrals, Moll met a specialized family of Jacobi polynomials. He conjectured that the corresponding coefficient sequences are log-concave. In this paper we settle Moll's conjecture by a nontrivial usage of computer algebra.
\end{abstract}

\section{INTRODUCTION}

Victor Moll begins his "personal story" [8] with the remark,

(...) It was even more of a surprise to discover that new things can still be said today about the mundane subject of integration of rational functions of a single variable and that this subject has connections with branches of contemporary mathematics as diverse as combinatorics, special functions, elliptic curves, and dynamical systems.

In this article we want to add another essential ingredient to his story, namely computer algebra. In Section 2 we will show how recently developed procedures can be used to retrieve observations which in Moll's original approach were derived with classical methods. In Section 3 we demonstrate that computer algebra can do even more, namely, by proving his log-concavity conjecture with a combination of various algorithms. In the Conclusion we briefly comment on a much stronger version of his conjecture.

\section{Setting the Stage}

Moll's starting point is the following double sum representation of a quartic integral.

Theorem 1. Let $a>-1$ and let $m$ be a natural number. Then

$$
\int_{0}^{\infty} \frac{1}{\left(x^{4}+2 a x^{2}+1\right)^{m+1}} d x=\frac{\pi}{2^{m+3 / 2}(a+1)^{m+1 / 2}} P_{m}(a),
$$

Received by the editors June 19, 2006.

2000 Mathematics Subject Classification. Primary 33F10, 05A20.

The first author was partially supported by FWF grants SFB F1305 and P16613-N12.

The second author was partially supported by FWF grant SFB F1301.

(C)2007 American Mathematical Society Reverts to public domain 28 years from publication 
where

$$
P_{m}(a)=\sum_{j, k}\left(\begin{array}{c}
2 m+1 \\
2 j
\end{array}\right)\left(\begin{array}{c}
m-j \\
k
\end{array}\right)\left(\begin{array}{c}
2 k+2 j \\
k+j
\end{array}\right) \frac{(a+1)^{j}(a-1)^{k}}{2^{3(k+j)}} .
$$

The proof is elementary and employs Wallis' integral formula; details are given in [2, Thm. 7.2.1]. Obviously, Theorem 1 gives a description of the quartic, which for specific $m$ can be computed in a finite number of steps. The remainder of Moll's article 8 discusses how further information about the family of polynomials $P_{m}(a)$ can be obtained. For instance, expanding $P_{m}(a)$ as

$$
P_{m}(a)=\sum_{l=0}^{m} d_{l}(m) a^{l}
$$

inspection quickly leads to the following positivity conjecture on the coefficients.

Proposition 1. For $0 \leq l \leq m$, we have $d_{l}(m)>0$.

2.1. $P_{m}(a)$ and Ramanujan's Master Theorem. Moll found a remarkable proof of Prop. 1. Namely, he was able to reformulate the problem in such a way that Ramanujan's Master Theorem could be invoked to prove the following alternative representation for the $P_{m}(a)$.

Theorem 2. Let $m$ be a natural number. Then

$$
P_{m}(a)=2^{-2 m} \sum_{k} 2^{k}\left(\begin{array}{c}
2 m-2 k \\
m-k
\end{array}\right)\left(\begin{array}{c}
m+k \\
k
\end{array}\right)(a+1)^{k} .
$$

For further details, see [2, Thm. 7.9.1]1 Obviously, Theorem 2 immediately implies positivity of the coefficients, i.e., the correctness of Proposition 11. In particular, after applying the binomial theorem one obtains the following single sum representation for the $d_{l}(m)$.

Proposition 2. For $l, m \geq 0$,

$$
d_{l}(m)=2^{-2 m} \sum_{k=l}^{m} 2^{k}\left(\begin{array}{c}
2 m-2 k \\
m-k
\end{array}\right)\left(\begin{array}{c}
m+k \\
k
\end{array}\right)\left(\begin{array}{l}
k \\
l
\end{array}\right) .
$$

In addition, a reformulation of the sum representation (2) allows us to identify the $P_{m}(a)$ within the mathematical knowledge base. Namely, rewriting it into standard hypergeometric format, $P_{m}(a)=2^{-2 m}\left(\begin{array}{c}2 m \\ m\end{array}\right){ }_{2} F_{1}\left(-m, m+1 ; \frac{1}{2}-m ; \frac{a+1}{2}\right)$, reveals that the $P_{m}(a)$ are nothing but special instances of Jacobi polynomials; i.e.,

$$
P_{m}(a)=P_{m}^{(\alpha, \beta)}(a) \quad \text { with } \alpha=m+\frac{1}{2} \text { and } \beta=-\left(m+\frac{1}{2}\right) .
$$

2.2. Positivity using computer algebra. In this subsection we demonstrate that computer algebra allows us to prove Proposition 1 in a completely routine fashion.

To this end we represent $d_{l}(m)$ as a triple sum,

$$
d_{l}(m)=\sum_{j, s, k} \frac{(-1)^{k+j-l}}{2^{3(k+s)}}\left(\begin{array}{c}
2 m+1 \\
2 s
\end{array}\right)\left(\begin{array}{c}
m-s \\
k
\end{array}\right)\left(\begin{array}{c}
2(k+s) \\
k+s
\end{array}\right)\left(\begin{array}{c}
s \\
j
\end{array}\right)\left(\begin{array}{c}
k \\
l-j
\end{array}\right)
$$

\footnotetext{
${ }^{1}$ In [1, identity (2) is derived directly from the integral representation in Thm. 1 via change of variables and table look-up.
} 
which is immediate from (11); see Theorem 7.3.1 in [2] after replacing $s$ by $s-j$ and then $k$ by $k+s$. Note that despite summing over all integers $j, s, k$ the sum is finite owing to the finite supports of the binomials involved.

The first step is to derive with the RISC package MultiSum [10] a suitable recurrence for the $d_{l}(m)$. MultiSum is an implementation of Wegschaider's algorithm, which is an extension of multivariate WZ summation [11. After loading the package into Mathematica by

$\ln [1]:=<<$ MultiSum.m

MultiSum Package by Kurt Wegschaider (enhanced by Axel Riese and Burkhard Zimmermann) - (C) RISC Linz - V2.02 $\beta$ (02/21/05)

we input the summand of the triple sum (5):

$$
\begin{aligned}
\ln [2]:=f= & (-1)^{k+j-l} / 2^{3(k+s)} \operatorname{Binomial}[2 m+1,2 s] \operatorname{Binomial}[m-s, k] \\
& \text { Binomial }[2(k+s), k+s] \operatorname{Binomial}[s, j] \operatorname{Binomial}[k, l-j]
\end{aligned}
$$

With the next command we determine candidate sets of shifts possibly arising in recurrences for $f=: F_{l, m, k, s, i}$ :

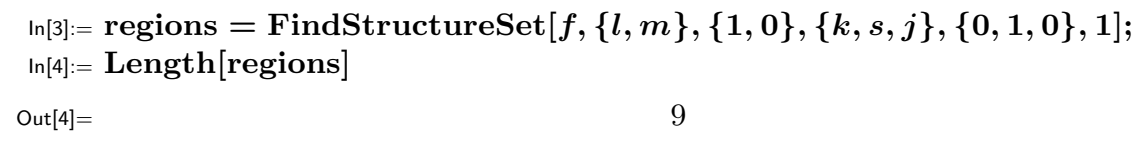

This means that we obtain nine such candidate sets. For the second one in this list we compute a summand recurrence as follows:

$$
\begin{aligned}
& \ln [5]:=\text { FindRecurrence }[f,\{l, m\},\{k, s, j\}, \text { regions [[2]], 1, WZ } \rightarrow \text { True] } \\
& \text { Out }[5]=\quad\left\{-4(l+m) F_{l-1, m, k-1, s-1, j-1}-2(2 l+4 m+3) F_{l, m, k-1, s-1, j-1}\right. \\
& +4(m+1) F_{l, m+1, k-1, s-1, j-1} \\
& =\Delta_{j}\left((2 k+6 s-7) F_{l-1, m, k-1, s-1, j-1}+2(2 l+4 m+3) F_{l, m, k-1, s-1, j-1}\right. \\
& \left.-4(m+1) F_{l, m+1, k-1, s-1, j-1}\right)+\Delta_{k}\left((-2 k+4 l+4 m-6 s+7) F_{l-1, m, k-1, s-1, j-1}\right. \\
& -4(k-l+s) F_{l-1, m, k, s-1, j-1} \\
& +(-2 k+4 l+4 m-2 s+7) F_{l, m, k-1, s-1, j}+4(k+m+2 s) F_{l, m, k-1, s, j} \\
& \left.-4(k-l) F_{l, m, k, s-1, j}-4(m+1) F_{l, m+1, k-1, s, j}\right) \\
& +\Delta_{s}\left((2 k+6 s-7) F_{l-1, m, k-1, s-1, j}+(2 k+4 m+2 s-1) F_{l, m, k-1, s-1, j}\right. \\
& \left.\left.-4(m+1) F_{l, m+1, k-1, s-1, j}\right)\right\}
\end{aligned}
$$

In the output, $\Delta_{i}$ denotes the forward difference operator, e.g.,

$$
\Delta_{k}\left((k-l+s) F_{l, m, k, s, j}\right)=(k+1-l+s) F_{l, m, k+1, s, j}-(k-l+s) F_{l, m, k, s, j} .
$$

Note: Since $F_{l, m, k, s, j}$ is hypergeometric w.r.t. each of the variables, any shift of $F_{l, m, k, s, j}$ is a rational function multiple of $F_{l, m, k, s, j}$. Consequently, after dividing both sides by $F_{l, m, k, s, j}$ the correctness check of Out 5 reduces to checking an equality of rational functions. Since $f=F_{l, m, k, s, j}$ has finite support w.r.t. the variables $j, k$, and $s$, we can sum both sides of Out 5 to obtain a recurrence for the sum $d_{l}(m)=: \operatorname{SUM}(l, m)$. This is done automatically by

$\ln [6]:=$ SumCertificate[\%]

Out $[6]=\{-2(l+m) \operatorname{SUM}(l-1, m)+(-2 l-4 m-3) \operatorname{SUM}(l, m)+2(m+1) \operatorname{SUM}(l, m+1)=0\}$

In other words, we have that for $0 \leq l \leq m+1$,

$$
2(m+1) d_{l}(m+1)=2(l+m) d_{l-1}(m)+(2 l+4 m+3) d_{l}(m) .
$$

This recurrence implies positivity of the $d_{l}(m)$. 
2.3. Identifying $P_{m}(a)$ using computer algebra. In this subsection we demonstrate that computer algebra allows us to identify the $P_{m}(a)$ in a completely routine fashion. This means that tools like Ramanujan's Master Theorem can be completely avoided. (Note: The conditions that make the Master Theorem applicable are in general nontrivial to check.)

Continuing the session above, we now compute a recurrence purely in $l$ :

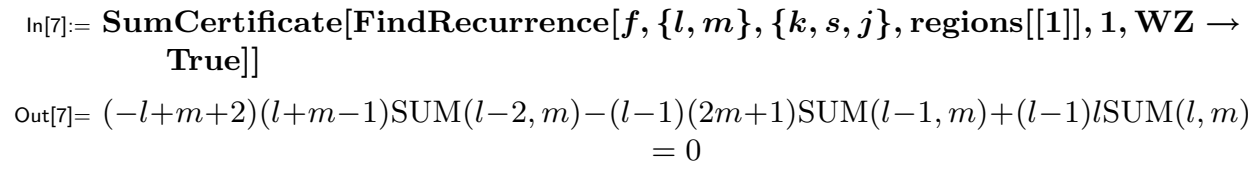

In other words, we have that for $0 \leq l \leq m+1$,

(7) $-(-2+l-m)(-1+l+m) d_{l-2}(m)-(l-1)(2 m+1) d_{l-1}(m)+(l-1) l d_{l}(m)=0$.

Note that in contrast to (6) this does not imply positivity of the $d_{l}(m)$. However, it enables us to identify $P_{m}(a)$ as a special instance of Jacobi polynomials. To this end, using the RISC package GeneratingFunctions [7] we convert (7) to a differential equation for $P_{m}(a)$, which is nothing but the ordinary generating function of the $d_{l}(m)$ for fixed $m$.

After loading the package into Mathematica by

$\ln [8]:=<<$ GeneratingFunctions.m

GeneratingFunctions Package by Christian Mallinger - (c) RISC Linz - V 0.68 $(07 / 17 / 03)$

we first input the recurrence (7)

$$
\begin{aligned}
\ln [9]:=\operatorname{rec} & =(-(-2+l-m)(-1+l+m) d[-2+l] \\
& -(-1+l)(1+2 m) d[-1+l]+(-1+l) l d[l]==0) ;
\end{aligned}
$$

The conversion to a differential equation for $P_{m}(a)=: P(a)$ is done automatically as follows:

$\ln [10]:=\operatorname{RE} 2 \mathrm{DE}[\mathrm{rec}, \boldsymbol{d}[l], \boldsymbol{P}[\boldsymbol{a}]]$

Out $[10]=$

$$
\left(m+m^{2}\right) P(a)-(1+2 a+2 m) P^{\prime}(a)-\left(a^{2}-1\right) P^{\prime \prime}(a)=0
$$

Finally we recall the differential equation for Jacobi polynomials $y(a):=P_{m}^{(\alpha, \beta)}(a)$,

$$
m(m+\alpha+\beta+1) y(a)-(\alpha-\beta+(\alpha+\beta+2) a) y^{\prime}(a)-\left(a^{2}-1\right) y^{\prime \prime}(a)=0,
$$

and compare the parameters,

$$
\begin{gathered}
\operatorname{In}[11]:=\operatorname{Solve}[\{\boldsymbol{\alpha}+\boldsymbol{\beta}=\mathbf{0}, \boldsymbol{\alpha}-\boldsymbol{\beta}=\mathbf{1}+\mathbf{2} \boldsymbol{m}, \boldsymbol{\alpha}+\boldsymbol{\beta}+\mathbf{2}=\mathbf{2}\},\{\boldsymbol{\alpha}, \boldsymbol{\beta}\}] \\
\text { Out }[11]= \\
\left\{\left\{\alpha \rightarrow \frac{1}{2}(2 m+1), \boldsymbol{\beta} \rightarrow-\frac{1}{2}-m\right\}\right\}
\end{gathered}
$$

Checking that $P_{m}(0), P_{m}^{\prime}(0)$ and the initial values $y(0), y^{\prime}(0)$ of $y(a)=P_{m}^{(\alpha, \beta)}(a)$ with $\alpha=m+\frac{1}{2}$ and $\beta=-\left(m+\frac{1}{2}\right)$ coincide, we can conclude (4).

Remarks.

(1) Owing to (5) the values $P_{m}(0)$ and $P_{m}^{\prime}(0)$ are hypergeometric double sums and thus can be evaluated with the package MultiSum.

(2) Taking the standard 3-term recurrence for Jacobi polynomials would fail since this one usually is derived under the assumption that the parameters $\alpha$ and $\beta$ are independent of $m$. 
2.4. Further recurrences for $d_{l}(m)$. In this subsection we compute further recurrences for $d_{l}(m)$ which will be needed in the next section. The MultiSum package would be powerful enough to continue to work with (15), but for the sake of simplicity we will use the representation (3).

$$
\begin{aligned}
& \operatorname{In}[12]:=\boldsymbol{g}=\mathbf{2}^{\boldsymbol{k - 2 m}} \operatorname{Binomial}[\mathbf{2} \boldsymbol{m}-\mathbf{2} \boldsymbol{k}, \boldsymbol{m}-\boldsymbol{k}] \text { Binomial }[\boldsymbol{m}+\boldsymbol{k}, \boldsymbol{k}] \text { Binomial }[\boldsymbol{k}, \boldsymbol{l}] \\
& \operatorname{In}[13]:=
\end{aligned}
$$

Note that here $g=F_{m, k}$. In other words, after replacing $m$ by $m+2$ we have for $0 \leq l \leq m+1$

$$
\begin{gathered}
4(l-m-2)(m+1)(m+2) d_{l}(m+2)=(l+m+1)(4 m+3)(4 m+5) d_{l}(m) \\
-2(m+1)\left(-4 l^{2}+8 m^{2}+24 m+19\right) d_{l}(m+1) .
\end{gathered}
$$

Finally, we derive another mixed recurrence, which is a counterpart to (6):

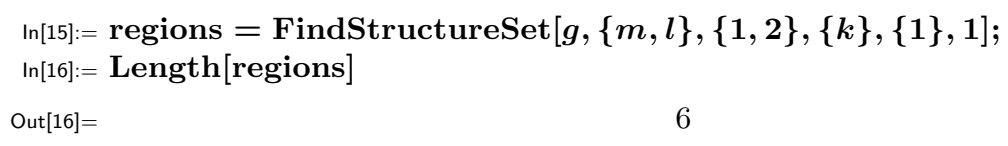

This means that we obtained six candidate regions for a mixed recurrence. For the second one in the list we compute a summand recurrence as follows:

$$
\begin{aligned}
& \operatorname{In}[17]:=\text { FindRecurrence }[g,\{m, l\},\{k\}, \text { regions }[[2]], 1, \mathrm{WZ} \rightarrow \text { True }] \\
& \text { Out }[17]= \\
& \left\{(2 l-4 m-5)(2 l-2 m-3)(l+m) F_{m, l-1, k}\right. \\
& +2(l-1) l(2 l-2 m-3) F_{m, l, k} \\
& -2(2 l-2 m-3)(l-m-2)(m+1) F_{m+1, l-1, k} \\
& =\Delta_{k}\left((2 l-2 m-3)(l+m)(-2 l+4 m+5) F_{m, l-1, k}\right. \\
& -2 l\left(2 l^{2}-2 m l-5 l-2 m^{2}-2 k-2 k m-2 m+1\right) F_{m, l, k} \\
& \left.\left.-2(m+1)\left(-2 l^{2}+4 m l+7 l-4 m^{2}+2 k+2 k m-11 m-8\right) F_{m+1, l-1, k}\right)\right\} \\
& \ln [18]:=\text { SumCertificate[\%] } \\
& \text { Out[18] }=\{(2 l-4 m-5)(l+m) \operatorname{SUM}(m, l-1)+2(l-1) l \operatorname{SUM}(m, l)-2(l-m-2)(m+1) \\
& \operatorname{SUM}(m+1, l-1)=0\}
\end{aligned}
$$

In other words, after replacing $l$ by $l+1$, we have for $0 \leq l \leq m$,

$$
\begin{aligned}
- & 2(2 l-4 m-3)(l+m+1) d_{l}(m)+4(l-m-1)(m+1) d_{l}(m+1) \\
& -4 l(l+1) d_{l+1}(m)=0 .
\end{aligned}
$$

\section{Proof of the Log-Concavity conjecture}

In addition to his positivity observation, Proposition 1, Moll observed that the sequence $d_{l}(m)$ is a candidate for being log-concave. The exact statement of his conjecture, proved below, is as follows. 
Theorem 3. For $0<l<m$, we have

$$
d_{l-1}(m) d_{l+1}(m) \leq d_{l}(m)^{2} .
$$

In this section, we prove this theorem by first deriving a sufficient condition on the sequence $d_{l}(m)$, which implies the log-concavity of $d_{l}(m)$. Then, we employ again computer algebra tools for proving this sufficient condition.

3.1. Derivation of a sufficient condition. In a first step, we use the recurrence equations (9) and (6) in order to eliminate the shifts in $l$ from (10) at the cost of introducing shifts in $m$. This gives the equivalent formulation

$$
\begin{gathered}
-\left(16 m^{3}+16 l m^{2}+40 m^{2}+28 l m+33 m+9 l+9\right) d_{l}(m)^{2} \\
-4(m+1)\left(2 l^{2}-4 m^{2}-7 m-3\right) d_{l}(m+1) d_{l}(m) \\
+4(l-m-1)(m+1)^{2} d_{l}(m+1)^{2} \leq 0 .
\end{gathered}
$$

Using Cylindrical Algebraic Decomposition (CAD) [4, 3, we compute in the second step an equivalent condition for the negation of (11), interpreting $m, l, d_{l}(m)$, and $d_{l}(m+1)$ as independent real variables $M, L, D_{0}, D_{1}$, respectively, and taking into account the knowledge $0<l<m$ and $d_{l}(m)>0$ (Prop. 1).

$$
\begin{aligned}
& \ln [19]:=\text { CylindricalDecomposition }\left[\left\{0<L<M, D_{0}>0, D_{1}>0,-\left(16 M^{3}\right.\right.\right. \\
& \left.+16 L M^{2}+40 M^{2}+28 L M+33 M+9 L+9\right) D_{0}^{2}-4(M+1)\left(2 L^{2}-4 M^{2}\right. \\
& \left.\left.-7 M-3) D_{1} D_{0}+4(L-M-1)(M+1)^{2} D_{1}^{2}>0\right\},\left\{M, L, D_{0}, D_{1}\right\}\right] \\
& M>\frac{1}{2}+\sqrt{2} \wedge \operatorname{Root}\left[4 \# 1^{3}-3 \# 1-4 M^{2}-4 M \&, 1\right]<L<M \wedge D_{0}>0 \\
& \wedge \frac{3-2 L^{2}+M(4 M+7)-\sqrt{L\left(4 L^{3}-3 L-4 M(M+1)\right)}}{2(M+1)(M-L+1)} D_{0}<D_{1} \\
& <\frac{3-2 L^{2}+M(4 M+7)+\sqrt{L\left(4 L^{3}-3 L-4 M(M+1)\right)}}{2(M+1)(M-L+1)} D_{0}
\end{aligned}
$$

In other words, we obtain that the formula

$$
0<l<m \wedge d_{l}(m)>0 \wedge d_{l}(m+1)>0 \wedge \neg \text { (11) }
$$

is equivalent over the reals to the formula

$$
m>\frac{1}{2}(1+2 \sqrt{2}) \wedge \operatorname{Root}\left(-4 m-4 m^{2}-3 X+4 X^{3}, 1\right)<l<m \wedge d_{l}(m)>0
$$

$$
\wedge \frac{p_{1}(l, m)-\sqrt{p_{2}(l, m)}}{p_{3}(l, m)} d_{l}(m)<d_{l}(m+1)<\frac{p_{1}(l, m)+\sqrt{p_{2}(l, m)}}{p_{3}(l, m)} d_{l}(m),
$$

where

$$
\begin{aligned}
& p_{1}(l, m)=3-2 l^{2}+m(4 m+7), \\
& p_{2}(l, m)=l\left(4 l^{3}-3 l-4 m(m+1)\right), \\
& p_{3}(l, m)=2(m+1)(m-l+1) .
\end{aligned}
$$

The symbol $\operatorname{Root}\left(-4 m-4 m^{2}-3 X+4 X^{3}, 1\right)$ denotes the algebraic function which maps $m$ to the smallest real root of the polynomial $-4 m-4 m^{2}-3 X+4 X^{3}$. The graph of this function is depicted in Figure 1 as a dotted curve. It divides the set of all points $(m, l), 0 \leq l \leq m$, under consideration into the sets

$$
S_{-}:=\left\{(m, l): 0<m \leq \frac{1}{2}+\sqrt{2} \vee 0<l \leq \operatorname{Root}\left(-4 m-4 m^{2}-3 X+4 X^{3}, 1\right)\right\}
$$

and

$$
S_{+}:=\left\{(m, l): m>\frac{1}{2}+\sqrt{2} \wedge \operatorname{Root}\left(-4 m-4 m^{2}-3 X+4 X^{3}, 1\right)<l<m\right\}
$$




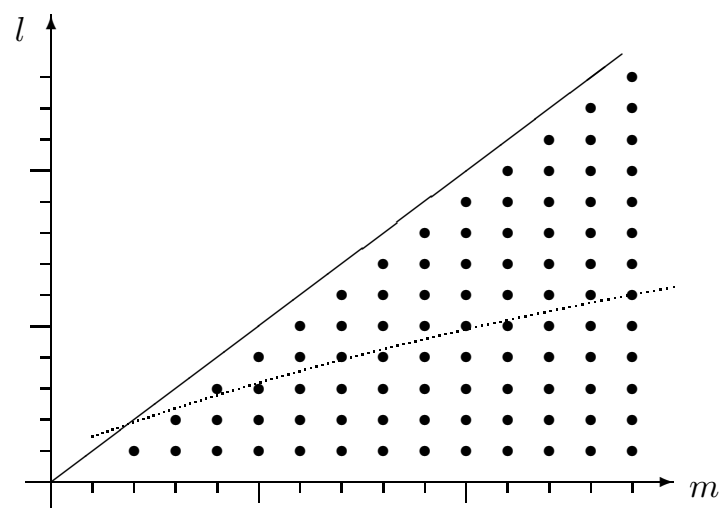

Figure 1. The points $(m, l)$ under consideration.

of points below and above this graph, respectively.

From the output (13), it follows that the log-concavity condition (10) cannot be violated at all for the points $(m, l) \in S_{-}$. In addition, for some point $(m, l) \in S_{+}$, it is violated if and only if

$$
\frac{p_{1}(l, m)-\sqrt{p_{2}(l, m)}}{p_{3}(l, m)} d_{l}(m)<d_{l}(m+1)<\frac{p_{1}(l, m)+\sqrt{p_{2}(l, m)}}{p_{3}(l, m)} d_{l}(m)
$$

for this point. In order to complete the proof for all $0<l<m$, it is therefore sufficient to show that

$$
d_{l}(m+1) \geq \frac{p_{1}(l, m)+\sqrt{p_{2}(l, m)}}{p_{3}(l, m)} d_{l}(m)
$$

for all $(m, l) \in S_{+}$, because then the violating condition (14) can never become true, and thus there does not exist any counterexample to the log-concavity condition for $0<l<m$.

For reasons to be explained in Section 3.2, we are not pleased with the square root expression $\sqrt{p_{2}(l, m)}$ in (15). It would be preferable to have a condition of the form $d_{l}(m+1) \geq \operatorname{rat}(l, m) d_{l}(m)$ for some rational function $\operatorname{rat}(l, m)$. In order to achieve this form, observe that it is sufficient to prove the modified condition

$$
d_{l}(m+1) \geq \frac{p_{1}(l, m)+\sqrt{p_{2}(l, m)+u(l, m)}}{p_{3}(l, m)} d_{l}(m)
$$

for a polynomial $u(l, m)$ which is nonnegative for $0<l<m$.

The idea is now to choose $u(l, m)$ in such a way that $p_{2}(l, m)+u(l, m)$ becomes a square. It turns out that $u(l, m)=4 l^{2}+4 l^{3}+4 l m(m+1)$ is a good choice, because $u(l, m) \geq 0$ and

$$
p_{2}(l, m)+u(l, m)=l^{2}(2 l+1)^{2} .
$$

Hence, (16) turns into the stronger condition

$$
d_{l}(m+1) \geq \frac{4 m^{2}+7 m+l+3}{2(m+1-l)(m+1)} d_{l}(m),
$$

which is stronger than (15) because $p_{3}(l, m)>0$ for the $l, m$ in question. 
Summarizing, if (17) is true for $(m, l) \in S_{+}$, then also (15) is true, and then (14) can never become true. But (14) is true for every possible counterexample to (11), so we are safe to conclude that no counterexample to (11) exists. Hence, since (11) is equivalent to (10), the log-concavity of $d_{l}(m)$ is established as soon as we have shown the boxed condition (17) above.

3.2. Proof of the sufficient condition. We now prove that (17) is true for all $0<l<m$. Let us denote this condition by $\Phi(l, m)$. The proof is by induction on $m$. We begin by showing the following induction step formula:

$$
\forall m \in \mathbb{N} \forall l \in \mathbb{N}:(0<l<m \wedge \Phi(l, m)) \Rightarrow \Phi(l, m+1) .
$$

This formula states that whenever $\Phi(l, m)$ is true for some bullet $(m, l)$ in Figure 1. then it is also true for its right neighboring bullet $(m+1, l)$. Applying the three term recurrence (8) to express $d_{l}(m+2)$ in terms of $d_{l}(m)$ and $d_{l}(m+1), \Phi(l, m+1)$ is found to be equivalent to

$$
d_{l}(m+1) \geq \frac{(m+l+1)(4 m+3)(4 m+5)}{2(m+1)(m+1-l)(4 m+4 l+5)} d_{l}(m) .
$$

Using, as earlier, real variables $M, L, D_{0}, D_{1}$ for representing $m, l, d_{l}(m), d_{l}(m+1)$, we can now prove the induction step formula by another application of CAD:

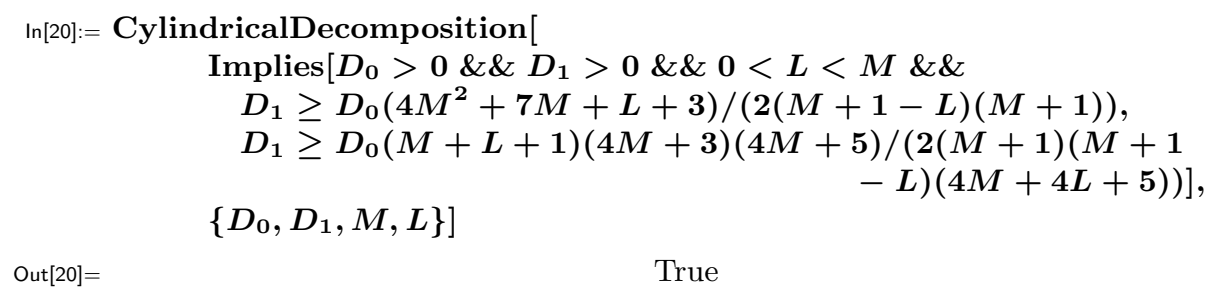

Out[20]=

True

This computation takes only fractions of a second of computation time, and it is in fact not hard to verify the statement by hand. We want to remark, however, that the computation time critically depends on the variable ordering supplied as the second argument to the CylindricalDecomposition command: If we had, for instance, chosen $\left\{M, L, D_{0}, D_{1}\right\}$ as the variable order, the computation would have taken more then forty minutes. But in either case, the computation returns "True" and this proves the induction step.

The proof is completed by showing, as the base of the induction, that $\Phi(l, m)$ is true for all the bullets $(l, m)$ right below the diagonal, i.e., that $\Phi(m, m+1)$ is true for all $m \geq 1$. Using the recurrences or the sum representations for $d_{l}(m)$, it is easy to obtain the special values

$$
\begin{gathered}
d_{m}(m+1)=2^{-m-2}(2 m+3)\left(\begin{array}{c}
2 m+2 \\
m+1
\end{array}\right) \\
d_{m}(m+2)=\frac{(m+1)\left(4 m^{2}+18 m+21\right)}{2^{m+4}(2 m+3)}\left(\begin{array}{c}
2 m+4 \\
m+2
\end{array}\right)
\end{gathered}
$$

(cf. Prop. 7.5.1 in [2]). Using these values, a straightforward computation now confirms that

$$
\Phi(m, m+1) \Longleftrightarrow \frac{d_{m}(m+2)}{d_{m}(m+1)} \geq \frac{2 m^{2}+8 m+7}{2(m+2)} \Longleftrightarrow \frac{m}{2(2 m+3)(m+2)} \geq 0,
$$

which is evident for $m \geq 1$. 
The method we applied for proving the truth of $\Phi(l, m)$ can be applied more generally for inequalities about quantities that can be defined via recurrence equations [5]. An implementation for univariate sequences that carries out the individual steps automatically is contained in the SumCracker package [6].

\section{Moll COnjeCtured MUCH MORE}

Define the operator $\mathcal{L}$ which maps a sequence $a_{l}$ to the sequence

$$
\mathcal{L} a_{l}:=a_{l-1} a_{l+1}-a_{l}^{2} .
$$

Then log-concavity of $a_{l}$ amounts to positivity of $\mathcal{L} a_{l}$. If the sequence $\mathcal{L} a_{l}$ is not only positive but also log-concave, then we say that $a_{l}$ is 2 -log-concave. In general, we say that $a_{l}$ is $k$-log-concave if $\mathcal{L}^{k} a_{l}$ is positive, and that $a_{l}$ is $\infty$-log-concave if $\mathcal{L}^{k} a_{l}$ is positive for every $k \geq 1$.

Moll has conjectured that the coefficients $d_{l}(m)$ are not only log-concave but even $\infty$-log-concave. This remains an open problem. Infinite log-concavity is open even for simpler sequences such as the binomial coefficient sequence $\left(\begin{array}{c}m \\ l\end{array}\right)$ (compare Project 7.9.3 in 2]). Here, we find that the $k$-log-concavity condition can always be brought into the form

$$
\operatorname{rat}_{k}(m, l)\left(\begin{array}{c}
m \\
l
\end{array}\right)^{\left(2^{k}\right)} \geq 0
$$

for some rational functions $\operatorname{rat}_{k}(m, l)$, which can be easily determined for each particular value of $k$. Applying again CAD, we were able to confirm that $\left(\begin{array}{c}m \\ l\end{array}\right)$ is $k$-log-concave for $k=0,1,2,3,4,5$. For $k \geq 6$ it is hopeless to apply CAD, because the complexity of this algorithm is sensitive towards the degree of the polynomials appearing in the inequalities, and the degrees of the numerator and denominator of $\operatorname{rat}_{k}(m, l)$ grow exponentially in $k$. For $k=6$, the degrees of the numerator and denominator are 133 and 196, respectively. As for the $\infty$-log-concavity, we do not see how currently available methods in symbolic computation could help in proving this conjecture.

Concerning the $k$-log-concavity of the original sequence $d_{l}(m)$, we have tried to apply the proof technique of Section 3 to establish 2-log-concavity, i.e.,

$$
\begin{aligned}
\left(d_{l}(m)^{2}-\right. & \left.d_{l-1}(m) d_{l+1}(m)\right)^{2} \\
& -\left(d_{l-1}(m)^{2}-d_{l-2}(m) d_{l}(m)\right)\left(d_{l+1}(m)^{2}-d_{l}(m) d_{l+2}(m)\right) \geq 0 .
\end{aligned}
$$

The recurrences (9) and (6) can again be used for obtaining an equivalent statement involving only shifts in $m$ but no shifts in $l$. This statement is polynomial in the $d_{l}(m+i)$ of degree 4 . As a consequence, the condition corresponding to (13) is much more complicated. It involves algebraic functions of degree up to 15, and it would require more than thirty pages to print it here. Under these circumstances, we have little hope that a proof of 2-log-concavity could be completed along these lines, not to mention that a human reader would have a hard time digesting it.

Note. All RISC software packages mentioned in this paper are available online at

$$
\text { http://www.risc.uni-linz.ac.at/research/combinat/software/ }
$$

\section{ACKNOWLEDGMENT}

We thank Carsten Schneider for computational support in using his package Sigma [9]. 


\section{REFERENCES}

[1] George Boros and Victor H. Moll. An integral hidden in Gradshteyn and Ryzhik. Journal of Computational and Applied Mathematics, 106:361-368, 1999. MR1696417 (2000c:33024)

[2] George Boros and Victor H. Moll. Irresistible Integrals. Cambridge University Press, Cambridge, 2004.

[3] Bob F. Caviness and Jeremy R. Johnson, editors. Quantifier Elimination and Cylindrical Algebraic Decomposition, Texts and Monographs in Symbolic Computation. Springer, 1998. MR.1634186 (99b:03007)

[4] George E. Collins. Quantifier elimination for the elementary theory of real closed fields by cylindrical algebraic decomposition. Lecture Notes in Computer Science, 33:134-183, 1975. MR0403962 (53:7771)

[5] Stefan Gerhold and Manuel Kauers. A procedure for proving special function inequalities involving a discrete parameter. In Proceedings of ISSAC'05, pages 156-162, 2005.

[6] Manuel Kauers. SumCracker: A package for manipulating symbolic sums and related objects. Journal of Symbolic Computation, 41:1039-1057, 2006. MR2251819

[7] Christian Mallinger. Algorithmic manipulations and transformations of univariate holonomic functions and sequences. Master's thesis, J. Kepler University, Linz, August 1996.

[8] Victor H. Moll. The evaluation of integrals: A personal story. Notices of the AMS, 49(3):311317, 2002. MR1879857 (2002m:11105)

[9] Carsten Schneider. The summation package Sigma: Underlying principles and a rhombus tiling application. Discrete Mathematics and Theoretical Computer Science, 6(2):365-386, 2004. MR2081481 (2005e:68270)

[10] Kurt Wegschaider. Computer generated proofs of binomial multi-sum identities. Master's thesis, RISC-Linz, May 1997.

[11] Herb S. Wilf and Doron Zeilberger. An algorithmic proof theory for hypergeometric (ordinary and $q$ ) multisum/integral identities. Invent. Math., 108:575-633, 1992. MR1163239 (93k:33010)

Research Institute for Symbolic Computation (RISC-Linz), Johannes Kepler UniverSITY Linz, AUstria

E-mail address: mkauers@risc.uni-linz.ac.at

Research Institute for Symbolic Computation (RISC-Linz), Johannes Kepler UniverSITY LinZ, AUstria

E-mail address: ppaule@risc.uni-linz.ac.at 